\title{
Disease Characteristics, Quality of Life, and Work Productivity by Enthesitis Site: Real-world Data From the US Corrona Psoriatic Arthritis/Spondyloarthritis Registry
}

\author{
Philip J. Mease ${ }^{1}$, Mei Liu², Sabrina Rebello², Winnie Hua², Robert R. McLean², Peter Hur ${ }^{3}$, \\ and Alexis Ogdie ${ }^{4}$
}

\begin{abstract}
Objective. To assess the effect of clinical enthesitis by body site in patients with psoriatic arthritis (PsA). Methods. Adults with PsA enrolled in the Corrona Psoriatic Arthritis/Spondyloarthritis Registry (March 2013-March 2018) were included. Enthesitis at enrollment was assessed by the Spondyloarthritis Research Consortium of Canada Enthesitis Index and classified by affected sites (upper, lower, or both). Disease activity (e.g., Clinical Disease Activity Index, Clinical Disease Activity Index for PsA), patient-reported outcomes (PRO; e.g., patient-reported pain and fatigue), and work productivity were compared between those with and without enthesitis using $t$ - or Wilcoxon rank-sum tests for continuous variables and chi-square or Fisher exact tests for categorical variables. The association of enthesitis with disease activity and PRO measures versus no enthesitis was modeled using multivariable-adjusted linear or logistic regression. Results. Of 2003 patients with PsA, 391 (19.5\%) had enthesitis: 80 (20.5\%) in upper sites only; 137 (35.0\%) in lower sites only; and 174 (44.5\%) in both. Regardless of location, disease activity and PRO were worse in patients with versus without enthesitis. In adjusted models, the presence of enthesitis at any site was significantly associated with worse disease activity versus no enthesitis. Enthesitis in lower or both upper and lower sites was associated with higher pain and fatigue scores and greater work impairment versus no enthesitis. Conclusion. Patients with clinical enthesitis had worse disease activity regardless of enthesitis location versus those without enthesitis, and patients with enthesitis in lower or both upper and lower sites had worse pain, fatigue, and work impairment.
\end{abstract}

Key Indexing Terms: epidemiology, psoriatic arthritis, quality of life, registries

Psoriatic arthritis (PsA) is an inflammatory rheumatic disease with diverse manifestations ${ }^{1}$. The Group for Research and Assessment of Psoriasis and Psoriatic Arthritis recognizes 6 clinical domains of PsA: peripheral disease, axial disease, enthesitis, dactylitis, skin disease, and nail disease; treatment guidelines recommend individualized strategies that improve disease activity in each domain ${ }^{2}$. Enthesitis, denoting inflammation at the insertion of tendons, ligaments, joint capsule fibers, or fascial insertion sites into bone ${ }^{3}$, is part of the Classification Criteria for Psoriatic Arthritis ${ }^{4}$ and is a hallmark of PsA ${ }^{2}$. Identifying enthesitis may be important for early diagnosis and subsequent treatment of $\mathrm{PsA}^{5,6}$.
This study was sponsored by Corrona, LLC. Corrona, LLC, has been supported through contracted subscriptions in the last 2 years by AbbVie, Amgen, Boehringer Ingelheim, Bristol Myers Squibb, Celgene, Crescendo, Eli Lilly and Company, Genentech, Gilead, GSK, Janssen, Merck, Momenta Pharmaceuticals, Novartis, Ortho Dermatologics, Pfizer Inc., Regeneron, Roche, Sun, and UCB. The design and conduct of the study were a collaborative effort between Corrona, LLC, and Novartis, and financial support for the study was provided by Novartis. Novartis participated in the interpretation of data and review and approval of the manuscript.

${ }^{I}$ P.J. Mease, MD, MACR, Swedish Medical Center/Providence St. Joseph Health and University of Washington, Seattle, Washington; ${ }^{2}$ M. Liu, PhD, S. Rebello, MPH, W. Hua, MS, R.R. McLean, DSc, MPH, Corrona, LLC, Waltham, Massachusetts; ${ }^{3}$. Hur, PharmD, MBA, Novartis Pharmaceuticals Corporation, East Hanover, New Jersey; ${ }^{4}$ A. Ogdie, MD, MCSE, Perelman School of Medicine at the University of Pennsylvania, Philadelphia,

Pennsylvania, USA.

PJM has received research grants from Celgene, Novartis, AbbVie, Amgen,
Bristol Myers Squibb, Eli Lilly, Pfizer, and UCB; consulting fees from Celgene, Corrona, Novartis, AbbVie, Amgen, Bristol Myers Squibb, Galapagos, Gilead, Janssen, Eli Lilly, Merck, Pfizer, Sun, and UCB; and speakers bureau fees from AbbVie, Amgen, Bristol Myers Squibb, Celgene, Genentech, Janssen, Pfizer, and UCB. M. Liu, S. Rebello, WH, and RRM are employees of Corrona, LLC.PH is an employee of Novartis Pharmaceuticals Corporation. AO has received consulting fees from Amgen, AbbVie, Bristol Myers Squibb, Celgene, Eli Lilly, Novartis, and Pfizer, and has received grant support from the National Institutes of Health/National Institute of Arthritis and Musculoskeletal and Skin Diseases, National Psoriasis Foundation, Rheumatology Research Foundation, Pfizer, and Novartis.

Address correspondence to Dr. P. Mease, Seattle Rheumatology Associates, 601 Broadway, Suite 600, Seattle, WA 98122, USA. Email:pmease@philipmease.com.

Full Release Article. For details see Reprints and Permissions at jrheum.org. Accepted for publication May 14, 2020. 
Enthesitis can lead to inflammatory and structural changes, thus causing pain and disability ${ }^{7}$ and is associated with nail psoriasis and dactylitis ${ }^{8,9}$. Patient factors associated with incident enthesitis include more actively inflamed joints, higher $\mathrm{BMI}$, and younger age at time of detection ${ }^{10}$. In several recent reports, the presence of enthesitis predicted poor PsA outcomes and may indicate a lower probability of achieving remission and low disease activity ${ }^{5,11}$. Presence of enthesitis in patients with PsA has been associated with axial and peripheral joint damage, a greater likelihood of developing joint ankyloses, overall higher disease activity, more pain, poorer quality of life (QOL) and functional status, sleep disturbance, and patient-reported pain and fatigue ${ }^{10,11,12}$.

While enthesitis is known to be associated with poor outcomes, little is known about the clinical effect of enthesitis by site of manifestation in patients with PsA. Enthesitis is more common in PsA than in other forms of rheumatic disease, including rheumatoid arthritis, and can aid in differential diagnosis of PsA; enthesitis has been observed in $\geq 35-50 \%$ of patients with PsA seen in clinical registries and up to $83 \%$ of patients in clinical trials 5 . In PsA, as in other forms of spondyloarthritis (SpA), enthesitis can occur in both the upper and lower extremities, but the latter seems to be more frequently involved-particularly the heel ${ }^{13,14}$. Reasons for the higher prevalence of enthesitis in lower extremities are unknown, although some studies have suggested that this may be due to the anatomy and mechanical load at these sites ${ }^{15,16}$. In a prospective, longitudinal cohort of Canadian patients with PsA, most patients had enthesitis involvement in the greater trochanter and Achilles tendon as well as the lateral and medial epicondyles, indicating that assessment of upper sites is also important when evaluating enthesitis ${ }^{10}$.

Understanding the effect of enthesitis by location may help inform physicians about important areas of focus for clinical evaluations. In this exploratory analysis, we therefore sought to compare disease activity and QOL between patients with PsA who have enthesitis at different locations (upper sites only, lower sites only, or both upper and lower sites) and those without enthesitis, and to assess the effect of enthesitis by site. As enthesitis in lower extremities may be more impactful on patients due to increased mechanical burden compared with upper sites, we hypothesized that patients with lower extremity involvement may experience worse disease activity and poorer QOL. Since imaging was not used to confirm the presence of enthesitis, our findings reflect the features and effect of clinical enthesitis.

\section{MATERIALS AND METHODS}

Data source. The Corrona Psoriatic Arthritis/Spondyloarthritis (PsA/ $\mathrm{SpA})$ Registry is a large, independent, prospective, observational cohort of patients diagnosed with PsA or SpA by a rheumatologist. Patients were recruited from 44 private and academic practice sites across 27 states in the USA, with 46 participating rheumatologists. As of June 2018, data on 3195 patients with PsA/SpA had been collected. The study protocol was approved by a central institutional review board (IRB; New England Independent Review Board, NEIRB No. 120160070) for private practice sites and the local governing IRB of participating academic sites. All registry subjects were required to provide written informed consent and authorization prior to participating.
Study design and study population. This is a cross-sectional study that included all adult patients with PsA enrolled in the Corrona PsA/SpA Registry between March 2013 and March 2018. Data were collected at the registry enrollment visit and included patient demographics (age, sex, race/ethnicity, marital status, and patient-reported work status), clinical characteristics (physician-reported history of comorbidities and disease and symptom duration), treatment profiles [prior and current biologic and conventional synthetic disease-modifying antirheumatic drug (csDMARD) use], and disease activity measures [modified minimal disease activity (MDA), tender joint count (TJC), swollen joint count (SJC), Spondyloarthritis Research Consortium of Canada (SPARCC) Enthesitis Index, dactylitis count, Clinical Disease Activity Index (CDAI), proportion of affected body surface area (BSA), Disease Activity Index for Psoriatic Arthritis (DAPSA), clinical DAPSA (cDAPSA), and physician global assessment]. The modified MDA for this study excluded the tender entheseal point criterion for MDA and was defined as meeting $\geq 5$ of the following criteria: TJC $\leq 1, \mathrm{SJC} \leq 1, \mathrm{BSA} \leq 3 \%$, patient pain visual analog scale $(\mathrm{VAS}) \leq 15$, patient global assessment $(\mathrm{PtGA}) \mathrm{VAS} \leq 20$, and Health Assessment Questionnaire-Disability Index (HAQ-DI) $\leq 0.5^{17}$. QOL measures [PtGA VAS, patient-reported pain and fatigue, HAQ-DI, EuroQol Quality of Life VAS scale (EQ-VAS), EuroQol 5-dimension scale (EQ-5D)] and work productivity measures [Work Productivity and Activity Impairment (WPAI) questionnaire] were also included.

Enthesitis assessment. Patients were classified as having clinically defined enthesitis at any site using the SPARCC Enthesitis Index, a measure of enthesitis based on the presence of tenderness at 18 entheseal sites (Figure 1$)^{18}$. Tenderness is recorded as present (1) or absent (0) at each site. The prevalence of enthesitis at each site among patients with enthesitis is listed as a total percentage of the left and right affected sites; patients may have reported enthesitis at $>1$ site. For scoring purposes, the inferior patella and tibial tuberosity are considered 1 site because of their anatomical proximity. The overall score ranges from 0 to 16 ; a score of 0 indicates the absence of enthesitis and higher scores indicate a greater burden of enthesitis. Patients with enthesitis were further classified by the location of affected sites: upper sites only (medial epicondyle, lateral epicondyle, and supraspinatus insertion into the greater tuberosity of the humerus), lower sites only (greater trochanter, quadriceps tendon insertion into superior border of patella, patellar ligament insertion into inferior pole of patella or tibial tubercle, Achilles tendon insertion into calcaneum, and plantar fascia insertion into calcaneum), and both upper and lower sites.

Data analysis. Means and frequencies of characteristics in all patients with enthesitis and each enthesitis site group (upper, lower, and both) were compared with those in patients without enthesitis (reference group) using chi-square tests or Fisher exact tests for categorical variables and $t$-tests or Wilcoxon rank-sum tests for continuous variables. The Spearman rank-order correlation test was used to determine the correlation of SPARCC Enthesitis Index scores with disease activity, QOL, and WPAI measures. Linear regression coefficients $(\beta)$ for continuous variables and logistic regression OR for categorical variables were calculated to estimate the multivariable-adjusted association of enthesitis at different sites (upper, lower, or both) with clinical and patient-reported outcomes relative to no enthesitis. Models were adjusted for age, sex, race, BMI, marital status, duration of PsA, depression, nail psoriasis, fibromyalgia (FM), history of csDMARD use, history of biologic use, and current csDMARD use. The covariates adjusted in the multivariable models included a list of a priori variables (e.g., age, sex, race, and BMI) and variables identified through significance testing $(P<0.05)$ in the baseline tables (e.g., marital status, duration of PsA, depression, nail psoriasis, fibromyalgia, history of csDMARD use, history of biologic use, and current csDMARD use). Due to the overlap of sites assessed by the SPARCC Enthesitis Index and tender points assessed in FM, a sensitivity analysis to exclude patients identified as having FM at registry enrollment was performed to examine the relationship between enthesitis and disease activity measures. 

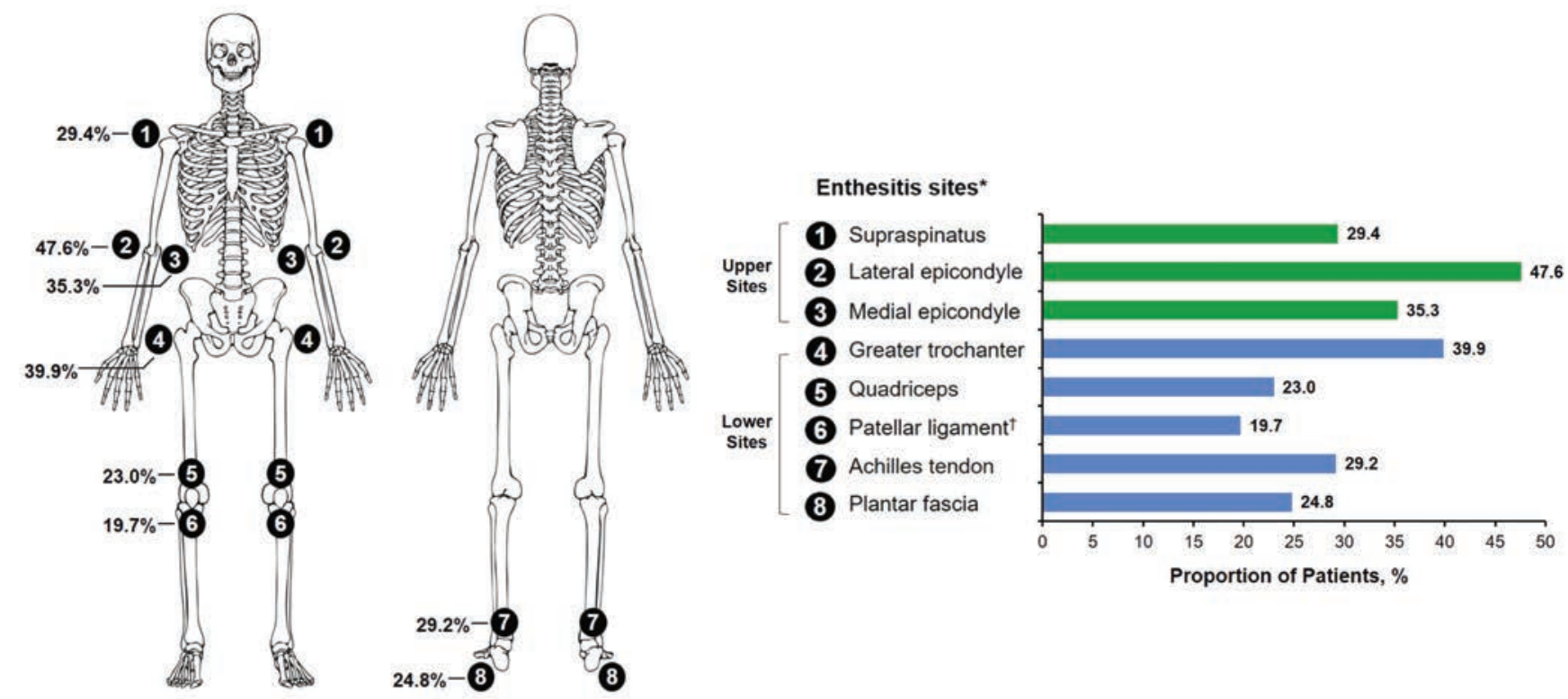

Figure 1. Prevalence of enthesitis by individual enthesitis site among patients with enthesitis $(\mathrm{N}=391) .{ }^{*}$ Percentages listed are a total of the left and right affected sites; patients may have reported enthesitis at $>1$ site. ${ }^{\dagger}$ For scoring purposes, the inferior patellar and tibial tuberosity are considered 1 site because of their anatomical proximity.

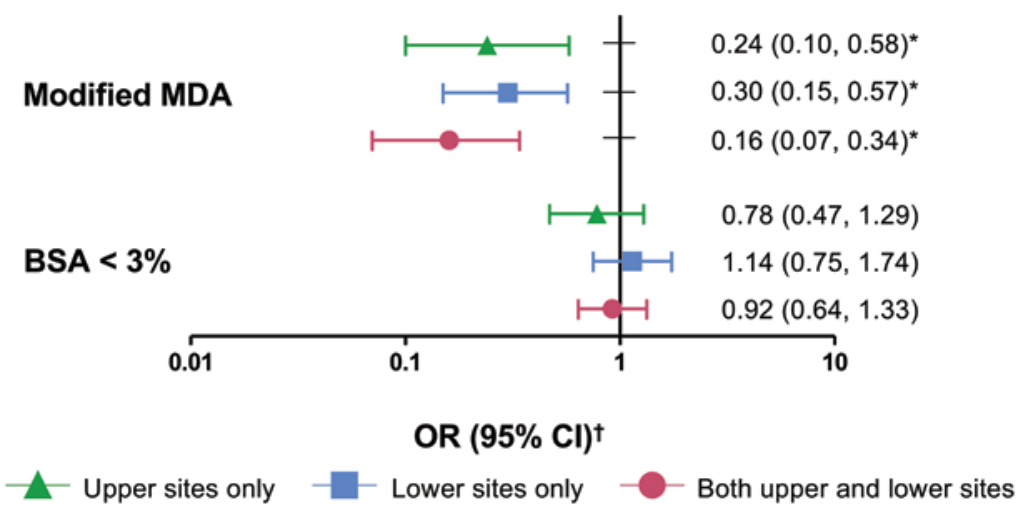

Figure 2. Likelihood (OR) of having modified MDA and BSA $<3 \%$ in groups with enthesitis in the upper extremities only, lower extremities only, and both upper and lower extremities relative to no enthesitis among patients with PsA in the Corrona PsA/SpA Registry. ${ }^{*} P<0.05$. ${ }^{\dagger}$ Analyzed using logistic regression models adjusted for age, sex, race, BMI, marital status, duration of PsA, depression, nail psoriasis, fibromyalgia, history of csDMARD use, history of biologic use, and current csDMARD use. BSA: body surface area; csDMARD: conventional synthetic disease-modifying antirheumatic drug; MDA: modified minimal disease activity; PsA: psoriatic arthritis; SpA: spondyloarthritis.

\section{RESULTS}

Patient characteristics at enrollment. A total of 2003 patients with PsA were included in the analyses, of whom 391 (19.5\%) had a positive SPARCC score consistent with enthesitis at enrollment. Of the 391 patients with enthesitis, 80 (20.5\%) had enthesitis in upper sites only, 137 (35.0\%) in lower sites only, and 174 (44.5\%) in both upper and lower sites (Table 1). The prevalence of enthesitis at each site among patients with enthesitis is shown in Figure 1.

Compared with those without enthesitis, patients with enthesitis had a similar symptom duration (11.4 vs 10.8 yrs) but a shorter mean disease duration $(8.7$ vs 7.0 yrs; $P<0.05)$ regardless of enthesitis site (Table 1). Patients affected in the lower sites only and both upper and lower sites were more likely to be female $(P<0.05)$. All enthesitis groups had a greater prevalence of FM versus the group with no enthesitis; patients with enthesitis in upper sites only and in both upper and lower sites also had a greater prevalence of nail psoriasis, and those with enthesitis in lower sites only had a greater prevalence of depression versus those without enthesitis $(P<0.001$; Table 1$)$. The proportions of patients currently receiving biologics or csDMARD were 
Table 1. Demographics, clinical characteristics, and treatment profiles of patients with PsA in the Corrona PsA/SpA Registry at their enrollment visit, stratified by location of SPARCC enthesitis sites.

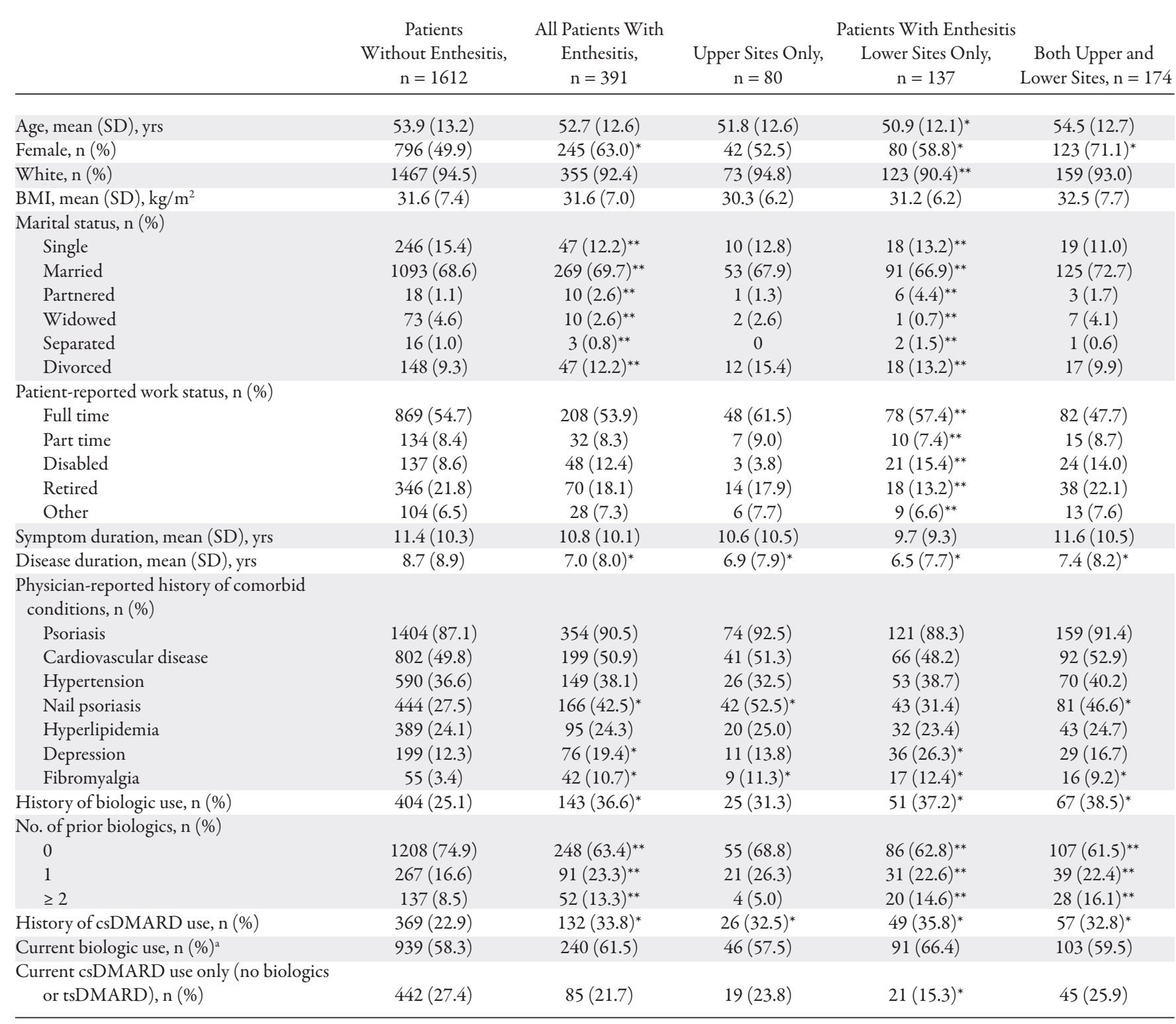

${ }^{a}$ Not including tsDMARD. ${ }^{*} P<0.05$ for comparison between the overall or location enthesitis groups and the no enthesitis group. ${ }^{* *} P<0.05$ for comparison of overall distribution across categories between the overall or location enthesitis groups and the no enthesitis group. csDMARD: conventional synthetic disease-modifying antirheumatic drug; PsA: psoriatic arthritis; SpA: spondyloarthritis; SPARCC: Spondyloarthritis Research Consortium of Canada; tsDMARD: targeted synthetic disease-modifying antirheumatic drug.

comparable between patients with enthesitis in upper sites only or both upper and lower sites and those without enthesitis (Table 1). Regardless of location of enthesitis sites, patients with enthesitis had worse disease activity, QOL, and work and activity impairment at enrollment for most measures compared with patients without enthesitis $(P<0.05$; Table 2$)$.

After excluding patients with FM, 1906 patients were eligible for the sensitivity analysis. Of these, $349(18.3 \%)$ had enthesitis and 1557 (81.7\%) had no enthesitis. Of those with enthesitis, 71 (20.3\%) had enthesitis at upper sites, $120(34.4 \%)$ at lower sites, and $158(45.3 \%)$ in both upper and lower sites. Demographics, clinical characteristics, and treatment profiles of patients without FM at their enrollment visit, stratified by location of SPARCC enthesitis sites, are shown in Supplementary Table 1 (available with the online version of this article). Compared with those without enthesitis, a higher proportion of patients with enthesitis were female and patients with enthesitis had a shorter disease duration and were more likely to have a history of nail psoriasis, depression, uveitis, and psoriasis $(P<0.001)$. Patients with enthesitis also were more likely to have a history of prior biologic and targeted synthetic DMARD and csDMARD use compared with those without enthesitis. Regardless of location 
Table 2. Disease activity, QOL, and work productivity of patients with PsA in the Corrona PsA/SpA Registry at their enrollment visit, stratified by location of SPARCC enthesitis sites.

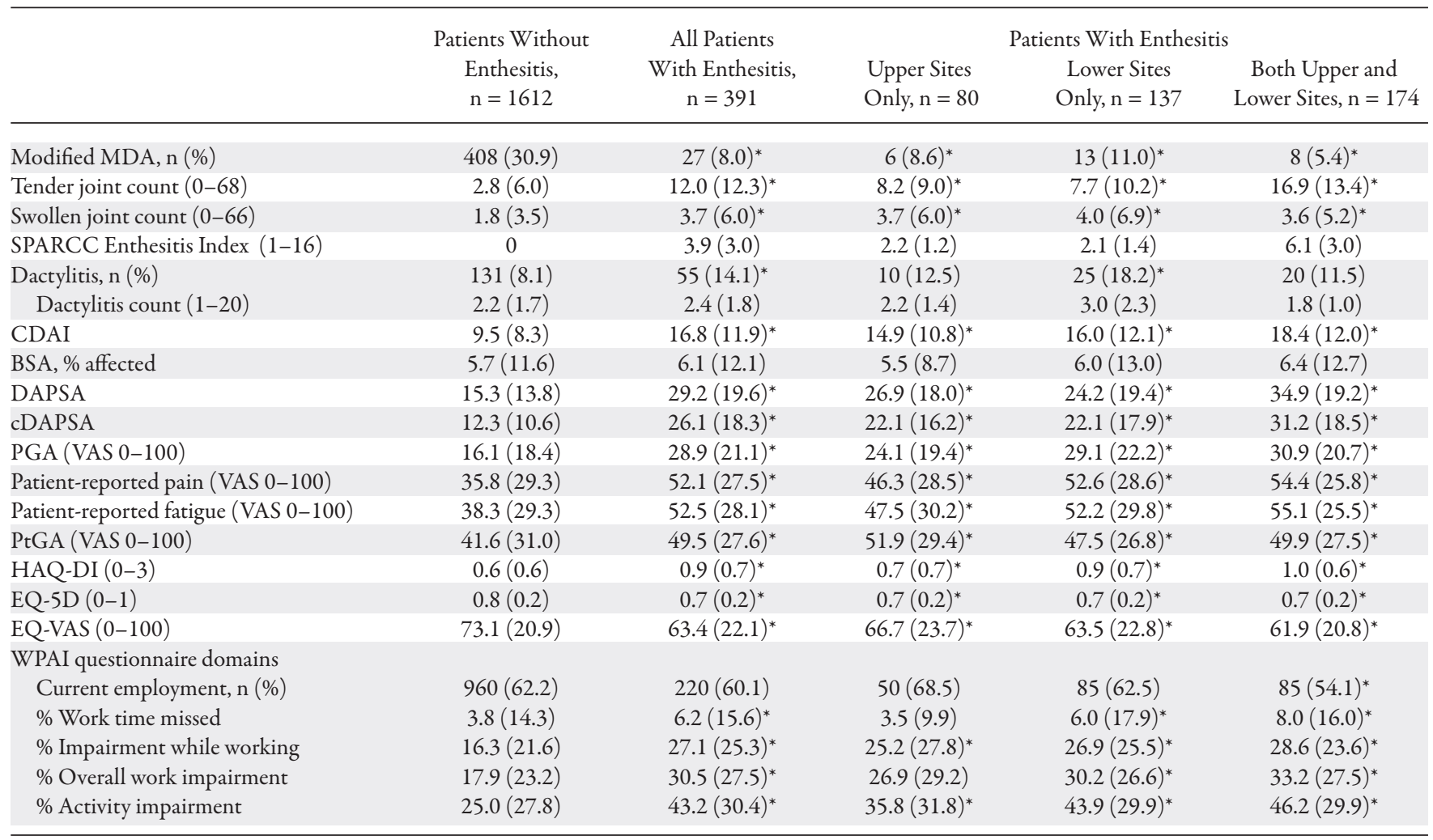

All values are presented as mean $(\mathrm{SD})$ unless otherwise stated. ${ }^{*} P<0.05$ for comparison between the overall or body location between the enthesitis groups and the no enthesitis group. BSA: body surface area; CDAI: Clinical Disease Activity Index; cDAPSA: clinical Disease Activity Index for Psoriatic Arthritis; DAPSA: Disease Activity Index for Psoriatic Arthritis; EQ-5D: Euroqol 5-dimension scale; EQ-VAS: EuroQol Quality of Life visual analog scale; HAQ-DI: Health Assessment Questionnaire-Disability Index; MDA: minimal disease activity (defined as meeting $\geq 5$ of the following criteria: tender joint count $\leq 1$, swollen joint count $\leq 1, \mathrm{BSA} \leq 3 \%$, patient pain VAS $\leq 15$, PtGA VAS $\leq 20$, and HAQ-DI $\leq 0.5$ ); PGA: physician global assessment; PsA: psoriatic arthritis; PtGA: patient global assessment; QOL: quality of life; SpA: spondyloarthritis; SPARCC: Spondyloarthritis Research Consortium of Canada; VAS: visual analog scale; WPAI: Work Productivity and Activity Impairment.

of enthesitis sites, patients with enthesitis had worse disease activity and QOL at enrollment for most measures compared with patients without enthesitis $(P<0.05$; Supplementary Table 2, available with the online version of this article).

SPARCC enthesitis site correlation. Overall, the SPARCC Enthesitis Index score was significantly correlated with higher disease activity and worse QOL (Table 3). Weak positive correlations as measured by the Spearman correlation coefficient were observed between the SPARCC Enthesitis Index score and CDAI (0.272), HAQ-DI (0.237), DAPSA (0.391), pain (0.230), fatigue (0.201), and PtGA (0.117) scores and with percentages of work and activity impairment (0.191-0.253). A negative correlation was observed between the SPARCC Enthesitis Index score and modified continuous modified MDA score $(-0.311)$.

As with the primary analysis, a mild positive correlation was observed between the SPARCC Enthesitis Index score and CDAI, HAQ-DI, DAPSA, pain, fatigue, PtGA, and all 4 WPAI domains as measured by the Spearman correlation coefficient (Supplementary Table 3, available with the online version of this article). Mild negative correlations were similarly found between SPARCC count and modified continuous modified MDA and EQ-5D.
Multivariable-adjusted association of enthesitis site by location with disease activity, QOL, and WPAI. In multivariable-adjusted models, the presence of enthesitis at any location was associated with a lower likelihood of modified MDA (Figure 2). The presence of enthesitis at any location was also associated with higher CDAI, cDAPSA, and PtGA scores versus the absence of enthesitis (Figure 3). Presence of enthesitis in lower sites only or in both upper and lower sites was associated with significantly higher pain and fatigue scores and with greater work and activity impairment versus absence of enthesitis (Figure 3). For several outcomes, there was a trend toward increasing impact by location of enthesitis sites (lowest to highest: upper sites only, lower sites only, both upper and lower sites) on disease activity (CDAI and CDAPSA), QOL (pain, fatigue, and HAQ-DI), and work productivity and activity impairment, although the CI were overlapping (Figure 3).

\section{DISCUSSION}

In this real-world population of patients with PsA, overall disease severity was greater in patients with clinical enthesitis, at any site, than in those without enthesitis. Although some 
Table 3. Spearman correlation coefficients of SPARCC score with disease activity, QOL, and WPAI measures.

\begin{tabular}{|c|c|c|c|}
\hline Variables & $\mathrm{N}$ & Spearman Correlation Coefficient & $P$ \\
\hline CDAI & 1852 & 0.272 & $<0.001$ \\
\hline HAQ-DI & 1855 & 0.237 & $<0.001$ \\
\hline DAPSA & 1000 & 0.391 & $<0.001$ \\
\hline Modified MDA (total score) ${ }^{a}$ & 1659 & -0.311 & $<0.001$ \\
\hline Patient-reported pain & 1843 & 0.230 & $<0.001$ \\
\hline Patient-reported fatigue & 1962 & 0.201 & $<0.001$ \\
\hline Patient global assessment & 1961 & 0.117 & $<0.001$ \\
\hline \multicolumn{4}{|l|}{ WPAI } \\
\hline \% Work time missed & 979 & 0.135 & $<0.001$ \\
\hline \% Impairment while working & 1036 & 0.191 & $<0.001$ \\
\hline$\%$ Overall work impairment & 914 & 0.206 & $<0.001$ \\
\hline$\%$ Activity impairment & 1735 & 0.253 & $<0.001$ \\
\hline
\end{tabular}

a This variable is the total score of the 6 criteria for modified MDA: tender joint count $\leq 1$, swollen joint count $\leq 1$, $\mathrm{BSA} \leq 3 \%$, patient pain VAS $\leq 15$, patient global assessment VAS $\leq 20$, and HAQ-DI $\leq 0.5$. BSA: body surface area; CDAI: Clinical Disease Activity Index; DAPSA: Disease Activity Index for Psoriatic Arthritis; HAQ-DI: Health Assessment Questionnaire-Disability Index; MDA: minimal disease activity (defined as meeting $\geq 5$ of the following criteria: tender joint count $\leq 1$, swollen joint count $\leq 1, \mathrm{BSA} \leq 3 \%$, patient pain VAS $\leq 15$, patient global assessment VAS $\leq 20$, and HAQ-DI $\leq 0.5$ ); QOL: quality of life; SPARCC: Spondyloarthritis Research Consortium of Canada; VAS: visual analog scale; WPAI: Work Productivity and Activity Impairment.

evidence suggests that lower extremity enthesitis would be more common due to mechanical loading ${ }^{19,20}$, the lateral epicondyles were among the most frequently affected sites in this population. There was a trend toward increasing disease severity by location of enthesitis sites (lowest to highest: upper sites only, lower sites only, both upper and lower sites) with respect to disease activity, QOL, and work productivity and activity impairment. These results suggest that while detecting enthesitis in both the upper and lower extremities is important for comprehensive assessment of disease severity, it may also be prudent to identify patients with enthesitis at specific sites. The relationship between enthesitis and disease activity measures remained unchanged when patients with FM were excluded from the sensitivity analysis, thus strengthening the association of clinical enthesitis with worse PsA disease severity.

Although the presence of enthesitis has been associated with worse disease activity and QOL in patients with PsA ${ }^{11,21,22,23,24,25,26}$, information on the effect of enthesitis by location on PsA disease activity, QOL, and work productivity is limited. One study of patients with SpA revealed that 3 of the 7 enthesitis sites examined (iliac crest, posterior spina iliaca, and Achilles tendon) were associated with work incapacity ${ }^{21}$. To our knowledge, our study was the first to assess the effect of enthesitis by location in US patients with PsA. In our study, we observed that the effect of enthesitis on overall disease severity may depend on the location of affected sites-there appeared to be a trend toward higher disease activity in lower sites when compared with upper sites, suggesting that lower sites may be an important area of focus. Enthesitis in both upper and lower sites was associated with greater disease severity than either upper or lower sites alone. Taken together, these results highlight the importance of evaluating both upper and lower sites for a comprehensive assessment of enthesitis and disease severity ${ }^{21}$.
The SPARCC Enthesitis Index was initially developed for the assessment of enthesitis in patients with $\mathrm{SpA}$ in general; during its development, investigators reported that patients with enthesitis had significantly greater Bath Ankylosing Spondylitis Disease Activity Index, Bath Ankylosing Spondylitis Functional Index, ASQOL index, and Short Form-36 health survey scores compared with those without enthesitis ${ }^{18}$. In PsA clinical studies, the SPARCC Enthesitis Index has demonstrated good responsiveness and construct validity ${ }^{27}$. We used the SPARCC Enthesitis Index in our study because it allows for enthesitis assessment of both peripheral and axial sites ${ }^{18}$ and has demonstrated reliability in both PsA and SpA ${ }^{27,28}$. However, because clinical indexes rely on the presence of entheseal tenderness and evaluate points that overlap with those assessed in FM, it may be challenging to distinguish between true enthesitis and entheseal tenderness due to central sensitization during clinical examinations ${ }^{29,30,31}$. Imaging modalities are also available for enthesitis detection. Although magnetic resonance imaging (MRI) has shown promise in detecting subclinical enthesitis ${ }^{32}$, ultrasound detection is often the method of choice for enthesitis imaging because it is more cost-effective than MRI and permits real-time clinical evaluation ${ }^{33}$.

Despite the increasing availability of imaging and clinical tools, enthesitis is often underdiagnosed in real-world clinical practice ${ }^{14}$. Because managing enthesitis is crucial to the overall control of PsA, it is important for clinicians to evaluate enthesitis using the clinical indexes or imaging tools available to them. Since imaging techniques may not be readily accessible by rheumatologists in real-world clinical practice, clinical examination of enthesitis may currently be more practical for patient evaluation. Patient input about body sites that contribute to these factors might help direct enthesitis assessments.

This study has some limitations that should be considered. 


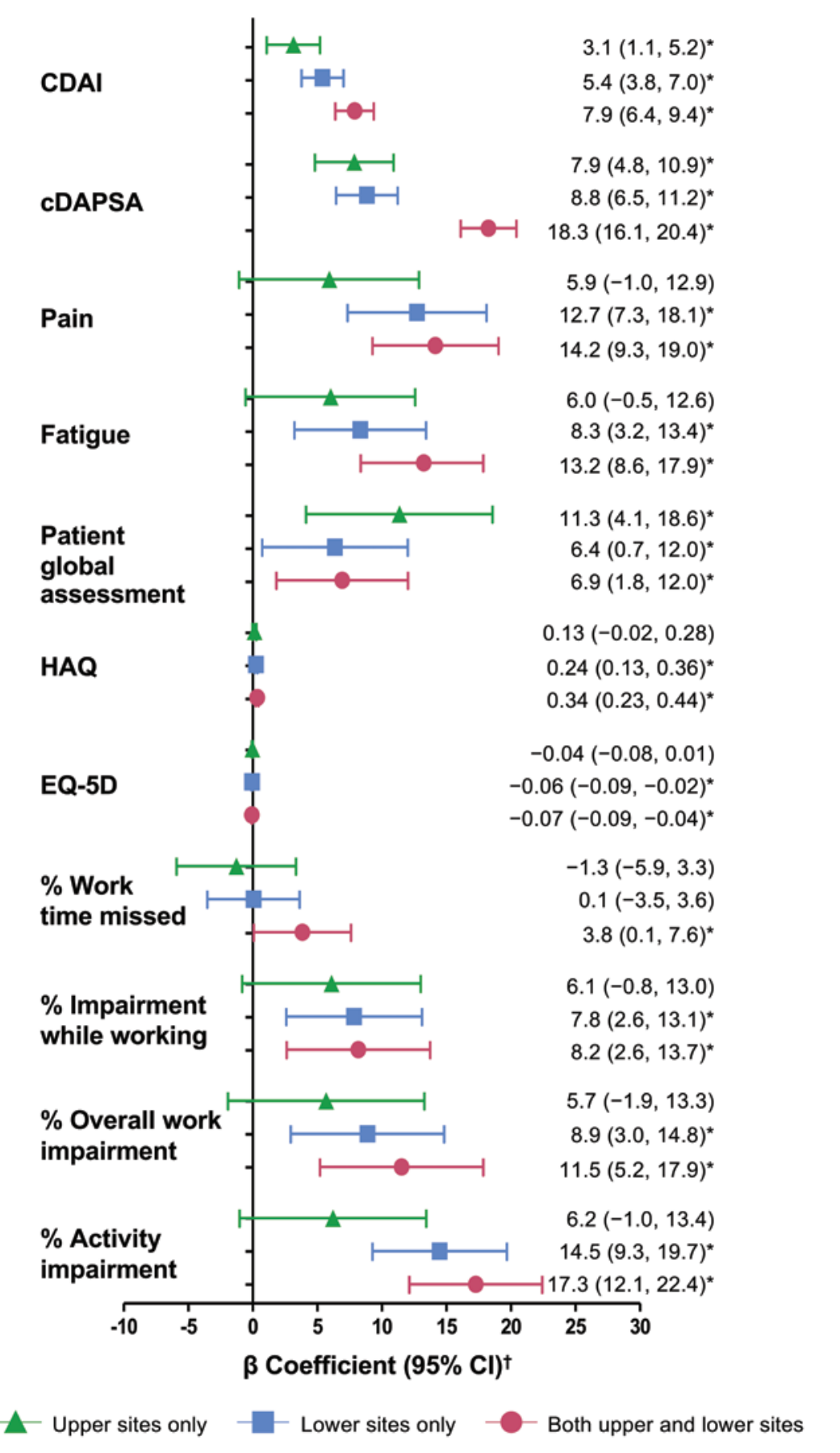

Figure 3. Association $(\beta)$ of having enthesitis in the upper extremities only, lower extremities only, and both upper and lower extremities with disease activity, QOL, and work productivity relative to no enthesitis among patients with PsA in the Corrona PsA/SpA Registry. ${ }^{*} P<0.05$. † Analyzed using linear regression models adjusted for age, sex, race, BMI, marital status, duration of PsA, depression, nail psoriasis, fibromyalgia, history of csDMARD use, history of biologic use, and current csDMARD use; $\beta$ coefficients are nonstandardized. CDAI: Clinical Disease Activity Index; cDAPSA: clinical Disease Activity Index for Psoriatic Arthritis; csDMARD: conventional synthetic disease-modifying antirheumatic drug; EQ-5D: 5-dimension EuroQol scale; HAQ: Health Assessment Questionnaire; PsA: psoriatic arthritis; QOL: quality of life; SpA: spondyloarthritis.

Patients in this study are routinely seen and treated by rheumatologists voluntarily participating in the Corrona PsA/SpA Registry and may not be representative of all US patients with
PsA. The lower prevalence of enthesitis in this cohort than in cohorts in other clinical registries ${ }^{5}$ may reflect the effects of prior effective treatment for PsA. Beyond the use of imaging, 
which may not be practical in daily clinical practice, an established and reliable method for identifying which patients have true enthesitis and which patients have entheseal point tenderness without inflammation is not currently available. As such, our findings reflect the features and effect of clinical enthesitis. Finally, generally having more sites of enthesitis is associated with greater disease burden. This could be an explanation for the greater effect of enthesitis at both sites compared with enthesitis in only upper or only lower sites. However, we are unable to adjust for the total number of sites in the comparison of upper to lower sites due to potential collinearity. Although it may seem obvious that enthesitis contributes to the overall assessment of disease activity, enthesitis is not frequently assessed in clinical practice. One of the key points of this manuscript is that, given the important contribution of enthesitis to overall disease activity, enthesitis should be routinely assessed.

In conclusion, our findings suggest that the presence of clinically defined enthesitis is associated with worse PsA disease severity, regardless of enthesitis location. In this real-world cohort, we observed that patients with enthesitis in lower sites or both upper and lower sites had worse pain, fatigue, and work impairment. Assessing enthesitis in both upper and lower sites may improve our understanding of the full scope of PsA disease activity. Further, management of enthesitis is important for ameliorating overall disease activity.

\section{ACKNOWLEDGMENT}

Support for third-party writing assistance for this manuscript, furnished by Kheng Bekdache, $\mathrm{PhD}$, of Health Interactions, Inc., was provided by Novartis Pharmaceuticals Corporation, East Hanover, New Jersey, USA.

\section{ONLINE SUPPLEMENT}

Supplementary material accompanies the online version of this article.

\section{REFERENCES}

1. Gladman DD. Clinical features and diagnostic considerations in psoriatic arthritis. Rheum Dis Clin North Am 2015;41:569-79.

2. Coates LC, Kavanaugh A, Mease PJ, Soriano ER, Acosta-Felquer ML, Armstrong AW, et al. Group for research and assessment of psoriasis and psoriatic arthritis 2015 treatment recommendations for psoriatic arthritis. Arthritis Rheumatol 2016;68:1060-71.

3. Ritchlin CT. Therapies for psoriatic enthesopathy. A systematic review. J Rheumatol 2006;33:1435-8.

4. Taylor W, Gladman D, Helliwell P, Marchesoni A, Mease P, Mielants $\mathrm{H}$, et al. Classification criteria for psoriatic arthritis: development of new criteria from a large international study. Arthritis Rheum 2006;54:2665-73.

5. Kaeley GS, Eder L, Aydin SZ, Gutierrez M, Bakewell C. Enthesitis: a hallmark of psoriatic arthritis. Semin Arthritis Rheum 2018; 48:35-43.

6. McGonagle D, Lories RJ, Tan AL, Benjamin M. The concept of a "synovio-entheseal complex" and its implications for understanding joint inflammation and damage in psoriatic arthritis and beyond. Arthritis Rheum 2007;56:2482-91.

7. Tan AL, McGonagle D. Psoriatic arthritis: correlation between imaging and pathology. Joint Bone Spine 2010;77:206-11.

8. Lai TL, Pang HT, Cheuk YY, Yip ML. Psoriatic nail involvement and its relationship with distal interphalangeal joint disease. Clin Rheumatol 2016;35:2031-7.
9. Raposo I, Torres T. Nail psoriasis as a predictor of the development of psoriatic arthritis. Actas Dermosifiliogr 2015;106:452-7.

10. Polachek A, Li S, Chandran V, Gladman DD. Clinical enthesitis in a prospective longitudinal psoriatic arthritis cohort: incidence, prevalence, characteristics, and outcome. Arthritis Care Res 2017;69:1685-91.

11. Mease PJ, Karki C, Palmer JB, Etzel CJ, Kavanaugh A, Ritchlin CT, et al. Clinical characteristics, disease activity, and patient-reported outcomes in psoriatic arthritis patients with dactylitis or enthesitis: results from the Corrona Psoriatic Arthritis/Spondyloarthritis Registry. Arthritis Care Res 2017;69:1692-9.

12. Polachek A, Cook R, Chandran V, Gladman DD, Eder L. The association between sonographic enthesitis and radiographic damage in psoriatic arthritis. Arthritis Res Ther 2017;19:189.

13. D’Agostino MA, Olivieri I. Enthesitis. Best Pract Res Clin Rheumatol 2006;20:473-86.

14. Kehl AS, Corr M, Weisman MH. Review: enthesitis: new insights into pathogenesis, diagnostic modalities, and treatment. Arthritis Rheumatol 2016;68:312-22.

15. Debusschere K, Cambré I, Gracey E, Elewaut D. Born to run: the paradox of biomechanical force in spondyloarthritis from an evolutionary perspective. Best Pract Res Clin Rheumatol 2017;31:887-94.

16. Lories RJ. Advances in understanding the pathophysiology of spondyloarthritis. Best Pract Res Clin Rheumatol 2018;32:331-41.

17. Coates LC, Fransen J, Helliwell PS. Defining minimal disease activity in psoriatic arthritis: a proposed objective target for treatment. Ann Rheum Dis 2010;69:48-53.

18. Maksymowych WP, Mallon C, Morrow S, Shojania K, Olszynski WP, Wong RL, et al. Development and validation of the Spondyloarthritis Research Consortium of Canada (SPARCC) Enthesitis Index. Ann Rheum Dis 2009;68:948-53.

19. Jacques P, Lambrecht $S$, Verheugen E, Pauwels E, Kollias G, Armaka $\mathrm{M}$, et al. Proof of concept: enthesitis and new bone formation in spondyloarthritis are driven by mechanical strain and stromal cells. Ann Rheum Dis 2014;73:437-45.

20. Lories RJ, Matthys P, de Vlam K, Derese I, Luyten FP. Ankylosing enthesitis, dactylitis, and onychoperiostitis in male DBA/ 1 mice: a model of psoriatic arthritis. Ann Rheum Dis 2004;63:595-8.

21. Carneiro S, Bortoluzzo A, Gonçalves C, Braga da Silva JA, Ximenes AC, Bértolo M, et al. Effect of enthesitis on 1505 Brazilian patients with spondyloarthritis. J Rheumatol 2013;40:1719-25.

22. Gezer O, Batmaz I, Sariyildiz MA, Sula B, Ucmak D, Bozkurt M, et al. Sleep quality in patients with psoriatic arthritis. Int J Rheum Dis 2017;20:1212-8.

23. Healy PJ, Helliwell PS. Measuring clinical enthesitis in psoriatic arthritis: assessment of existing measures and development of an instrument specific to psoriatic arthritis. Arthritis Rheum 2008;59:686-91.

24. Jajić Z, Rajnpreht I, Kovačić N, Lukić IK, Velagić V, Grubišić F, et al. Which clinical variables have the most significant correlation with quality of life evaluated by SF-36 survey in a Croatian cohort of patients with ankylosing spondylitis and psoriatic arthritis? Rheumatol Int 2012;32:3471-9.

25. Tezel N, Yilmaz Tasdelen O, Bodur H, Gul U, Kulcu Cakmak S, Oguz ID, et al. Is the health-related quality of life and functional status of patients with psoriatic arthritis worse than that of patients with psoriasis alone? Int J Rheum Dis 2015;18:63-9.

26. Turan Y, Duruöz MT, Cerrahoglu L. Relationship between enthesitis, clinical parameters and quality of life in spondyloarthritis. Joint Bone Spine 2009;76:642-7.

27. Mease PJ. Measures of psoriatic arthritis: Tender and Swollen Joint Assessment, Psoriasis Area and Severity Index (PASI), Nail Psoriasis Severity Index (NAPSI), Modified Nail Psoriasis Severity Index 
(mNAPSI), Mander/Newcastle Enthesitis Index (MEI), Leeds Enthesitis Index (LEI), Spondyloarthritis Research Consortium of Canada (SPARCC), Maastricht Ankylosing Spondylitis Enthesis Score (MASES), Leeds Dactylitis Index (LDI), Patient Global for Psoriatic Arthritis, Dermatology Life Quality Index (DLQI), Psoriatic Arthritis Quality of Life (PsAQOL), Functional Assessment of Chronic Illness Therapy-Fatigue (FACIT-F), Psoriatic Arthritis Response Criteria (PsARC), Psoriatic Arthritis Joint Activity Index (PsAJAI), Disease Activity in Psoriatic Arthritis (DAPSA), and Composite Psoriatic Disease Activity Index (CPDAI). Arthritis Care Res 2011;63 Suppl 11:S64-85.

28. Mease PJ, Coates LC. Considerations for the definition of remission criteria in psoriatic arthritis. Semin Arthritis Rheum 2018; 47:786-96.

29. Brikman S, Furer V, Wollman J, Borok S, Matz H, Polachek A, et al. The effect of the presence of fibromyalgia on common clinical disease activity indices in patients with psoriatic arthritis: a cross-sectional study. J Rheumatol 2016;43:1749-54.
30. Marchesoni A, Atzeni F, Spadaro A, Lubrano E, Provenzano G, Cauli A, et al. Identification of the clinical features distinguishing psoriatic arthritis and fibromyalgia. J Rheumatol 2012;39:849-55.

31. Marchesoni A, De Marco G, Merashli M, McKenna F, Tinazzi I, Marzo-Ortega $\mathrm{H}$, et al. The problem in differentiation between psoriatic-related polyenthesitis and fibromyalgia. Rheumatology 2018;57:32-40.

32. Poggenborg RP, Eshed I, Østergaard M, Sørensen IJ, Møller JM, Madsen OR, et al. Enthesitis in patients with psoriatic arthritis, axial spondyloarthritis and healthy subjects assessed by 'head-to-toe' whole-body MRI and clinical examination. Ann Rheum Dis 2015;74:823-9.

33. Patil P, Dasgupta B. Role of diagnostic ultrasound in the assessment of musculoskeletal diseases. Ther Adv Musculoskelet Dis 2012;4:341-55. 